\title{
Exploring The Molecular Mechanisms of Classical Hodgkin Lymphoma Through Bioinformatics Analysis
}

\section{Zhu Lili}

Fuyang Hospital of Anhui Medical University https://orcid.org/0000-0003-3962-7946

\section{Zhu YuKun}

Fuyang Hospital of Anhui Medical University

\section{Zhuangzhuang Tian}

Fuyang Hospital of Anhui Medical University

\section{Yongsheng Li}

Fuyang Hospital of Anhui Medical University

Liyu Cao ( $\sim$ caoliyufyyy@163.com )

Fuyang Hospital of Anhui Medical University https://orcid.org/0000-0003-1374-4714

\section{Research}

Keywords: $\mathrm{cHL}$, differentially expressed genes, function enrichment analysis, hub genes

Posted Date: June 11th, 2021

DOl: https://doi.org/10.21203/rs.3.rs-586729/v1

License: (9) This work is licensed under a Creative Commons Attribution 4.0 International License. Read Full License 


\section{Abstract \\ Background}

Classic Hodgkin lymphoma $(\mathrm{CHL})$ is the most common $\mathrm{HL}$ in the modern society. Although the treatment of $\mathrm{cHL}$ has made great progress, its molecular mechanisms have yet to be deciphered.

\section{Objectives}

The purpose of this study is to find out the crucial potential genes and pathways associated with cHL.

\section{Methods}

We downloaded the cHL microarray dataset (GSE12453) from Gene Expression Omnibus (GEO) database and to identify the differentially expressed genes (DEGs) between cHL samples and normal samples through the limma package in R. Then, gene ontology (GO) and Kyoto Encyclopedia of Genes and Genomes (KEGG) pathway enrichment analysis of DEGs were carried out. Finally, we constructed the protein-protein interaction network to screen out the hub genes using Search Tool for the Retrieval of Interacting Genes (STRING) database.

\section{Results}

We screened out 788 DEGs in the cHL dataset, such as BATF3, IER3, RAB13 and FCRL2. G0 functional enrichment analysis indicated that the DEGs were related with regulation of lymphocyte activation, secretory granule lumen and chemokine activity. KEGG pathway analysis showed that the genes enriched in Prion disease, Complement and coagulation cascades and Parkinson disease Coronavirus diseaseCOVID-19 pathway. Protein-protein interaction network construction identified 10 hub genes (IL6, ITGAM, CD86, FN1, MMP9, CXCL10, CCL5, CD19, IFNG, SELL, UBB) in the network.

\section{Conclusions}

In the present investigation, we identified several pathways and hub genes related to the occurrence and development of $\mathrm{cHL}$, which may provide an important basis for further research and novel therapeutic targets and prognostic indicators for $\mathrm{cHL}$.

\section{Background}

Hodgkin lymphoma $(\mathrm{HL})$ is a unique lymphoid neoplasm that accounts for $10 \%$ of all diagnosed lymphomas and has an increasing incidence in the world (1). CHL, the most common malignancy in 
children and adolescents is a major type of $\mathrm{HL}$ and represents about $95 \%$ of all cases of $\mathrm{HL}(2,3)$. According to the World Health Organization (WHO), cHL is divided into four types: lymphocyte-rich, lymphocyte depleted, nodular sclerosis and mixed cellularity. In histopathology, $\mathrm{cHL}$ is characterized by specific large atypical cells, Hodgkin Reed-Sternberg (HRS) cells, which are often surrounded by abundant reactive inflammatory immune cells including $T$ cells, B cells, macrophages, plasma cells, eosinophils, and additional immune cells (4). Although many patients with $\mathrm{cHL}$ can be cured through radiotherapy and chemotherapy, a considerable number of patients have progressed and became difficult to treat, that is relapsed or refractory $\mathrm{CHL}(5,6)$. Programmed death-ligand (PD-L)1 and PD-L2 are often overexpressed in HRS cells and the tumor microenvironment, due to the genetic amplification at chromosome 9p24.1 (7, 8). In recent years, the results of a number of clinical trials have confirmed that PD-1/PD-L1 inhibitors have shown high efficacy in cHL patients (9). Despite the clear efficacy of PD-1/PD-L1 inhibitors, some patients will experience accelerated tumor progression and adverse reactions after treatment. The ultimate reason for the unsatisfactory therapeutic effect is our ambiguity about the molecular mechanism of tumors and the ambiguity of the mechanism of immunotherapies. In order to have a deeper and more comprehensive understanding of the molecular mechanism of the pathogenesis of $\mathrm{cHL}$, in the current study, we screened out the pathways and hub genes involved $\mathrm{cHL}$, which provided further understanding the molecular mechanism and a new idea for the treatment $\mathrm{cHL}$ in the future.

\section{Materials And Methods}

\section{Dataset preparation and DEGs identification}

We downloaded the microarray dataset (GSE12453) from GEO (http://www.ncbi.nlm.nih.gov/geo/) database. The dataset of GSE12453 contains 25 normal B cells samples, $12 \mathrm{cHL}$ samples and 30 other types of lymphoma samples. In the current study, normal B cells and $\mathrm{CHL}$ samples were used for further analysis. The GSE12453 dataset was based on GPL570 platforms (HG-U133_Plus_2; Affymetrix Human Genome U133 Plus 2.0 Array). The affy package was utilized to preprocess and normalize the raw data under the $\mathrm{R}$ environment (version 4.0.5). Through the preprocessing of the R package, the GSE312887 sample did not meet the requirements and excluded from the following analysis. In this study, the limma package was applied to screen out DEGs in CHL (10) and the cut-off criteria was set as adjusted $\mathrm{P}<0.05$ and $|\log \mathrm{FC}|>3$.

\section{Functional Enrichment Analysis Of Degs}

To further explore the biological function of DEGs, GO and KEGG pathway enrichment analyses were performed using the clusterProfiler package in R. The $\mathrm{GO}$ terms were divided into biological processes (BP), cellular component (CC) and molecular function (MF) ontologies, shown in the Fig. 2 . Among the BP ontology, the results showed that the DEGs mainly enriched inneutrophil activation, neutrophil mediated immunity and neutrophil degranulation. Among the CC ontology, the genes were correlated with secretory granule lumen, cytoplasmic vesicle lumen and collagen-containing extracellular matrix. Among the MF 
ontology, the results were involved in chemokine activity, chemokine receptor binding and CCR chemokine receptor binding. As for the pathways, KEGG pathway analysis results indicated that DEGs are mainly focused on the pathways concentrated in Prion disease, Complement and coagulation cascades and Viral protein interaction with cytokine and cytokine receptor. In addition, we also found that the DEGs were enriched in the pathway of Coronavirus disease-COVID-19, which ravaged the world in just six months and had a profound impact on the world.

\section{Ppi Network Construction And Identification Of Hub Genes}

To identify the hub genes that play an important role in cHL, the online Search Tool for the Retrieval of Interacting Genes (STRING database, Version 11.0; http://string-db.org/) was used to build protein-protein interaction (PPI) network and a combined score of not $<0.7$ was considered significant (13). Cytoscape software (version 3.7.1; http://cytoscape.org/) was applied to visualize the PPI network $(14,15)$. The CytoHubba, Cytoscape plug-in, was utilized to analyze the hub genes through node degree in the network, and the top 10 genes with most interactions were informally referred to as hub genes.

\section{Validation Of Hub Genes}

To verify the hub genes obtained from PPI network, we used the online database, the Cancer Cell Line Encyclopedia Cancer Cell Line Encyclopedia (CCLE), which contains mRNA expression levels in robust multichip average log2 of various cancer cell lines, to analyze transcription levels of the 10 hub genes in cHL cell lines $(16,17)$.

\section{Results}

\section{Identification of DEGs}

To identify DEGs between Hodgkin lymphoma and normal samples, the GSE12453 dataset was obtained from NCBI GEO datasets and the Limma package was applied to analyze the DEGs. The results showed that a total of 788 DEGs were screened out, in which 462 genes were upregulated and 326 genes were downregulated. As is shown in Fig.1, of which the volcano plot indicates all the genes and the heatmap shows the 100 most changed genes.

\section{Functional enrichment analysis of DEGs}

To further explore the biological function of DEGs, GO and KEGG pathway enrichment analyses were performed using the clusterProfiler package in $\mathrm{R}$. The $\mathrm{GO}$ terms were divided into biological processes (BP), cellular component (CC) and molecular function (MF) ontologies, shown in the Fig. 2 . Among the BP ontology, the results showed that the DEGs mainly enriched inneutrophil activation, neutrophil mediated immunity and neutrophil degranulation. Among the CC ontology, the genes were correlated with secretory granule lumen, cytoplasmic vesicle lumen and collagen-containing extracellular matrix. Among the MF 
ontology, the results were involved in chemokine activity, chemokine receptor binding and CCR chemokine receptor binding. As for the pathways, KEGG pathway analysis results indicated that DEGs are mainly focused on the pathways concentrated in Prion disease, Complement and coagulation cascades and Viral protein interaction with cytokine and cytokine receptor. In addition, we also found that the DEGs were enriched in the pathway of Coronavirus disease-COVID-19, which ravaged the world in just six months and had a profound impact on the world.

\section{Identification and validation of DEGs}

To find out the hub genes in DEGs, we used STRING database to build PPI network and Cytoscape to visualize the network. As is shown in the results (Fig.3), we visualized the top 50 genes with the most node degree in the network. And then, CytoHubba was applied to identify the hub genes and 10 hub genes (IL6, ITGAM, CD86, FN1, MMP9, CXCL10, CCL5, CD19, IFNG, SELL, UBB) were screened out. As for the results, most of the hub genes have not been reported in $\mathrm{CHL}$. To validate the significance of the genes, we downloaded their mRNA expression data in verious $\mathrm{CHL}$ cell lines from CCLE database. The results indicated that IL6, CD86, FN1 and CCL5 were overexpressed in CHL cell lines and ITGAM, MMP9, CXCL10, CD19, IFNG and SELL underexpressed in CHL cell lines, shown in the Fig.4.

\section{Discussion}

In recent years, the research on the pathogenesis of $\mathrm{cHL}$ has made great progress, which provides a basis for the treatment of $\mathrm{cHL}(18-21)$. Many studies have shown that the immune microenvironment composed of abnormal HRS cells and their surrounding immune cells provides suitable conditions for tumor development (22-24). In addition, studies also have indicated that the malignant HRS cells have highly PD-L1 expression on the cell surface, which results in the suppression of immune response $(7,8)$. The high expression of PD-L1 in HRS cells and its microenvironment makes the PD-1/PD-L1 signaling pathway a new target for tumor immunotherapy (25-27). A variety of PD-1/PD-L1 blockers have been used in clinical trials, and the results have shown that immunotherapies have a good effect on relapsed or refractory $\mathrm{cHL}$. However, the immunotherapy also has adverse reactions after treatment, due to the limited understanding of the biology of immune checkpoint bockade in $\operatorname{cHL}(25,28,29)$. Therefore, indepth study of the molecular mechanism of cHL is very necessary. In the current study, we used bioinformatics methods to analyze the intrinsic pathogenesis of $\mathrm{cHL}$ and we got very meaningful results related to $\mathrm{cHL}$. Firstly, we screened out 788 DEGs closely related to the occurrence and development of $\mathrm{cHL}$. Secondly, the results of $\mathrm{GO}$ analysis suggested that $\mathrm{CHL}$-related genes were enriched in the proliferation, differentiation and activation of white blood cells, which was consistent with the known theories. Thirdly, pathway analysis indicated that the development of $\mathrm{CHL}$ was related to NF-kappa B signaling pathway, Complement and coagulation cascades and PD-L1 expression and PD-1 checkpoint pathway in cancer. Finally, we identified10 hub genes (IL6, ITGAM, CD86, FN1, MMP9, CXCL10, CCL5, CD19, IFNG, SELL, UBB), which may play a significant role in the $\mathrm{CHL}$. 
In the present study, the results of analysis indicated that leukocyte function, NF-kappa B signaling pathway and PD-1 checkpoint pathway played a major role in the biological process of $\mathrm{cHL}$, which has been proven by several existing studies $(27,30,31)$. The consistency between our analysis results and existing research results shows the credibility of our analysis. In addition, for the first time, our work found that the pathway of Coronavirus disease-COVID-19 is related to $\mathrm{CHL}$. Our results indicate that COVID-19 may affect the occurrence of cHL. Some of the hub genes we screened have been reported to be related to $\mathrm{cHL}$, and some are the first time we have discovered that they play a key role in $\mathrm{cHL}$.

\section{Conclusion}

In short, our work not only validated previous studies, but also discovered new pathways and core genes that may affect $\mathrm{cHL}$, which provides new ideas for future studies of $\mathrm{cHL}$ molecular mechanisms and the search for drug targets.

\section{List Of Abbreviations}

$\mathrm{BP}$

Biological process; CC:Cellular component; CCLE:Cancer Cell Line Encyclopedia Cancer Cell Line Encyclopedia; CHL:Classic Hodgkin lymphoma; DEGs:Differentially expressed genes;

GEO

Gene Expression Omnibus; HRS:Hodgkin Reed-Sternberg; PD-L:Programmed death-ligand; PPI:Proteinprotein interaction; WHO:World Health Organization

\section{Declarations}

\section{Ethics approval and consent to participate}

Not applicable.

\section{Consent for publication}

All authors approved this publication.

\section{Availability of supporting data}

All data analyzed during this work are included in this published article.

\section{Competing interests}

The authors declare that they have no competing interests.

\section{Funding}


This work was supported by the Anhui Provincial Natural Science Foundation of China (grant number: 1808085MH283).

\section{Authors' contributions}

LYC designed the study. LLZ and YKZ performed the bioinformatic analysis and wrote the manuscript. ZZT and YSL reviewed and revised the manuscript. All authors read and approved the final manuscript.

\section{Acknowledgements}

None.

\section{Authors' information}

This part includes full names and email addresses of all co-authors on this manuscript. Liyu Cao: caoliyufyyy@163.com; Lili Zhu: 15055558699@163.com; Yukun Zhu: zhuyukun@tmu.edu.cn; Zhuangzhuang Tian: tian039102@163.com; Yongsheng Li: 864772488@qq.com.

\section{References}

1. Ibrahim AS, Khaled HM, Mikhail NN, Baraka H, Kamel $\mathrm{H}$. Cancer incidence in egypt: results of the national population-based cancer registry program. J Cancer Epidemiol. 2014;2014:437971.

2. Eichenauer DA, Aleman BMP, Andre $M$, Federico $M$, Hutchings $M$, Illidge $T$, et al. Hodgkin lymphoma: ESMO Clinical Practice Guidelines for diagnosis, treatment and follow-up. Ann Oncol. 2018;29(Suppl 4):iv19-29.

3. Group AW, Ccm, Group AW. Italian cancer figures, report 2012: Cancer in children and adolescents. Epidemiol Prev. 2013;37(1 Suppl 1):1-225.

4. Sabattini E, Bacci F, Sagramoso C, Pileri SA. WHO classification of tumours of haematopoietic and lymphoid tissues in 2008: an overview. Pathologica. 2010;102(3):83-7.

5. Santoro A, Bonadonna G, Valagussa P, Zucali R, Viviani S, Villani F, et al. Long-term results of combined chemotherapy-radiotherapy approach in Hodgkin's disease: superiority of ABVD plus radiotherapy versus MOPP plus radiotherapy. J Clin Oncol. 1987;5(1):27-37.

6. Canellos GP, Anderson JR, Propert KJ, Nissen N, Cooper MR, Henderson ES, et al. Chemotherapy of advanced Hodgkin's disease with MOPP, ABVD, or MOPP alternating with ABVD. N Engl J Med. 1992;327(21):1478-84.

7. Roemer MG, Advani RH, Ligon AH, Natkunam Y, Redd RA, Homer H, et al. PD-L1 and PD-L2 Genetic Alterations Define Classical Hodgkin Lymphoma and Predict Outcome. J Clin Oncol. 2016;34(23):2690-7.

8. Green MR, Monti S, Rodig SJ, Juszczynski P, Currie T, O'Donnell E, et al. Integrative analysis reveals selective 9p24.1 amplification, increased PD-1 ligand expression, and further induction via JAK2 in 
nodular sclerosing Hodgkin lymphoma and primary mediastinal large B-cell lymphoma. Blood. 2010;116(17):3268-77.

9. Ansell SM. Targeting immune checkpoints in lymphoma. Curr Opin Hematol. 2015;22(4):337-42.

10. Ritchie ME, Phipson B, Wu D, Hu Y, Law CW, Shi W, et al. limma powers differential expression analyses for RNA-sequencing and microarray studies. Nucleic Acids Res. 2015;43(7):e47.

11. Yu G, Wang LG, Yan GR, He QY. DOSE: an R/Bioconductor package for disease ontology semantic and enrichment analysis. Bioinformatics. 2015;31(4):608-9.

12. Yu G, Wang LG, Han Y, He QY. clusterProfiler: an R package for comparing biological themes among gene clusters. OMICS. 2012;16(5):284-7.

13. Szklarczyk D, Morris JH, Cook H, Kuhn M, Wyder S, Simonovic M, et al. The STRING database in 2017: quality-controlled protein-protein association networks, made broadly accessible. Nucleic Acids Res. 2017;45(D1):D362-D8.

14. Su G, Morris JH, Demchak B, Bader GD. Biological network exploration with Cytoscape 3. Curr Protoc Bioinformatics. 2014;47:8 13 1-24.

15. Diaz-Montana JJ, Gomez-Vela F, Diaz-Diaz N. GNC-app: A new Cytoscape app to rate gene networks biological coherence using gene-gene indirect relationships. Biosystems. 2018;166:61-5.

16. Barretina J, Caponigro G, Stransky N, Venkatesan K, Margolin AA, Kim S, et al. Addendum: The Cancer Cell Line Encyclopedia enables predictive modelling of anticancer drug sensitivity. Nature. 2019;565(7738):E5-6.

17. Barretina J, Caponigro G, Stransky N, Venkatesan K, Margolin AA, Kim S, et al. The Cancer Cell Line Encyclopedia enables predictive modelling of anticancer drug sensitivity. Nature. 2012;483(7391):603-7.

18. Connors JM, Cozen W, Steidl C, Carbone A, Hoppe RT, Flechtner HH, et al. Hodgkin lymphoma. Nat Rev Dis Primers. 2020;6(1):61.

19. Brockelmann PJ, Goergen H, Keller U, Meissner J, Ordemann R, Halbsguth TV, et al. Efficacy of Nivolumab and AVD in Early-Stage Unfavorable Classic Hodgkin Lymphoma: The Randomized Phase 2 German Hodgkin Study Group NIVAHL Trial. JAMA Oncol. 2020;6(6):872-80.

20. Fisher DAC, Oh ST. Unraveling the Architecture of Classic Hodgkin Lymphoma One Cell at a Time. Cancer Discov. 2020;10(3):342-4.

21. Aoki T, Chong LC, Takata K, Milne K, Hav M, Colombo A, et al. Single-Cell Transcriptome Analysis Reveals Disease-Defining T-cell Subsets in the Tumor Microenvironment of Classic Hodgkin Lymphoma. Cancer Discov. 2020;10(3):406-21.

22. Patel SS, Weirather JL, Lipschitz M, Lako A, Chen PH, Griffin GK, et al. The microenvironmental niche in classic Hodgkin lymphoma is enriched for CTLA-4-positive T cells that are PD-1-negative. Blood. 2019;134(23):2059-69.

23. Connors JM. Hodgkin lymphoma: outsmarting HRS cells. Blood. 2020;136(21):2362-4. 
24. Sanchez-Aguilera A, Montalban C, de la Cueva P, Sanchez-Verde L, Morente MM, Garcia-Cosio M, et al. Tumor microenvironment and mitotic checkpoint are key factors in the outcome of classic Hodgkin lymphoma. Blood. 2006;108(2):662-8.

25. Connors JM. Immunotherapy for older patients with classic Hodgkin lymphoma. Lancet Haematol. 2020;7(11):e776-e7.

26. Nie J, Wang C, Liu Y, Yang Q, Mei Q, Dong L, et al. Addition of Low-Dose Decitabine to Anti-PD-1 Antibody Camrelizumab in Relapsed/Refractory Classical Hodgkin Lymphoma. J Clin Oncol. 2019;37(17):1479-89.

27. Ansell SM, Lesokhin AM, Borrello I, Halwani A, Scott EC, Gutierrez M, et al. PD-1 blockade with nivolumab in relapsed or refractory Hodgkin's lymphoma. N Engl J Med. 2015;372(4):311-9.

28. Bair SM, Mato A, Svoboda J. Immunotherapy for the Treatment of Hodgkin Lymphoma: An Evolving Paradigm. Clin Lymphoma Myeloma Leuk. 2018;18(6):380-91.

29. Ansell SM. Immunotherapy in Hodgkin Lymphoma: The Road Ahead. Trends Immunol. 2019;40(5):380-6.

30. Brune MM, Juskevicius D, Haslbauer J, Dirnhofer S, Tzankov A. Genomic Landscape of Hodgkin Lymphoma. Cancers (Basel). 2021;13(4).

31. Thomas RK, Re D, Wolf J, Diehl V. Part I. Hodgkin's lymphoma-molecular biology of Hodgkin and Reed-Sternberg cells. Lancet Oncol. 2004;5(1):11-8.

\section{Figures}


A

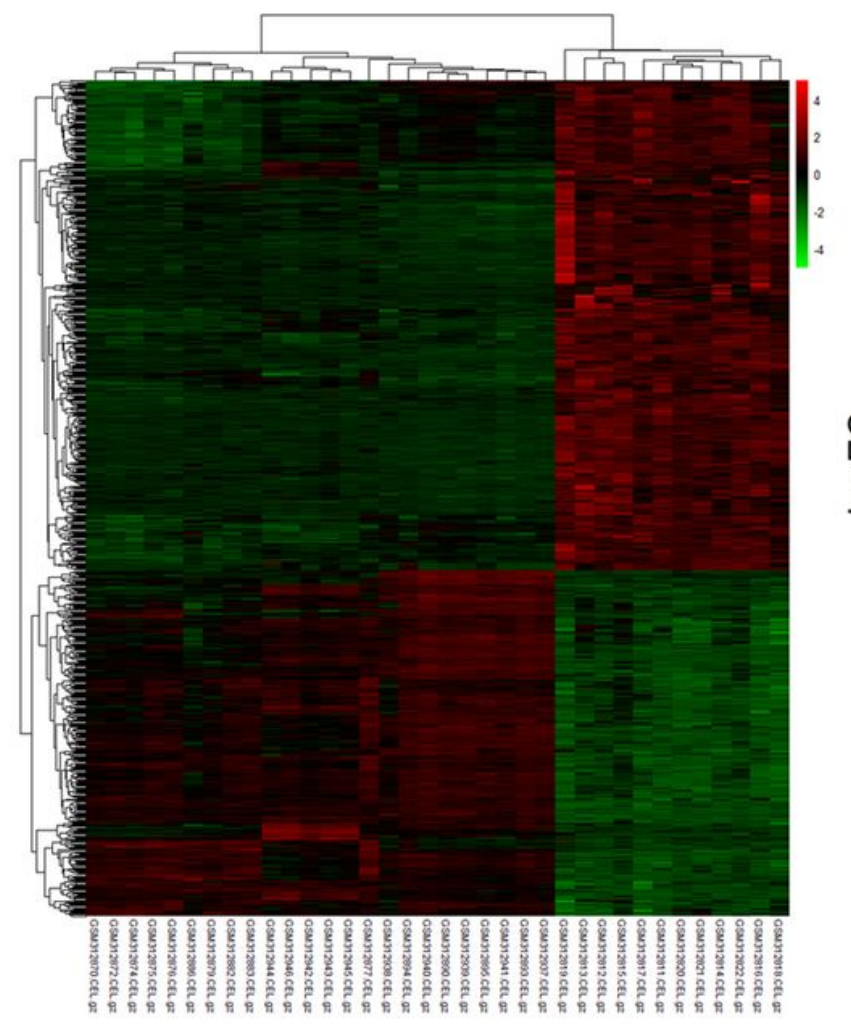

B

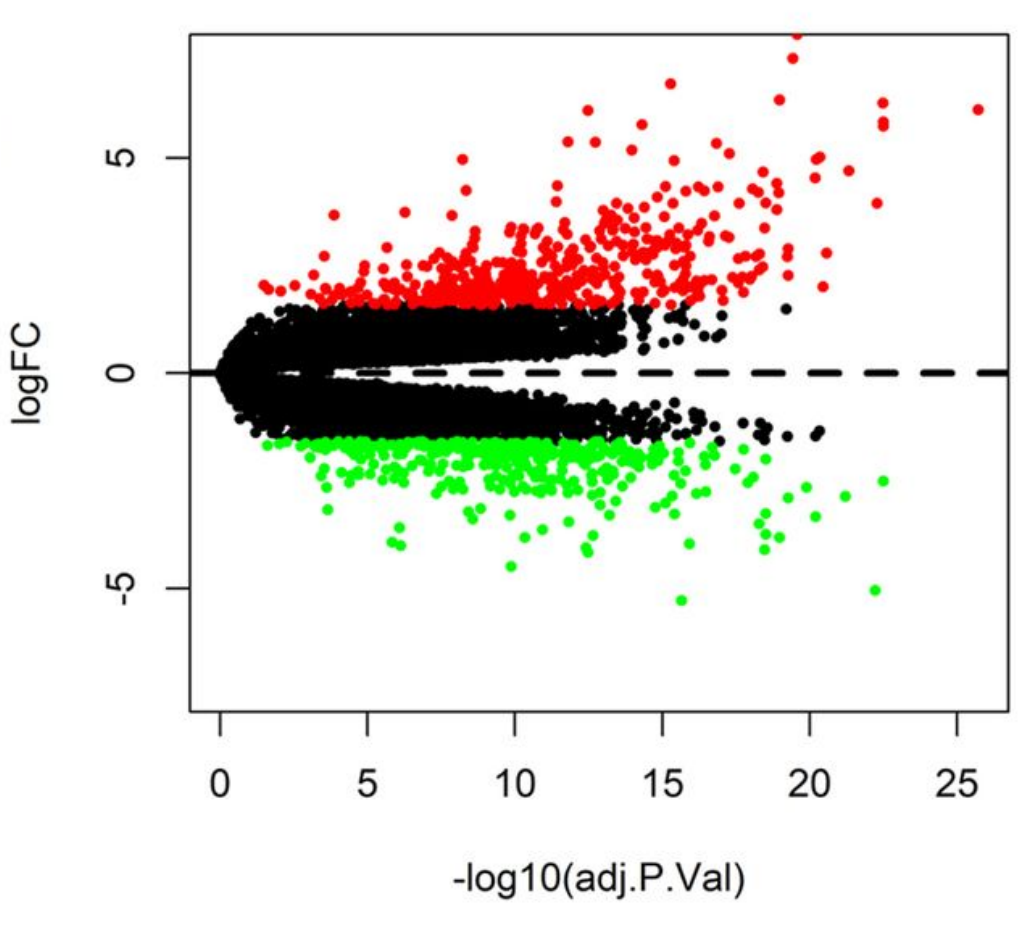

\section{Figure 1}

The differentially expressed genes of $\mathrm{cHL}$. (A). Volcano plot of the aberrant genes of between the $\mathrm{cHL}$ and normal samples. Red dots indicate significantly upregulated genes. Green dots indicate significantly downregulated genes. (B). Heat map hierarchical clustering indicates DEGs in GSE12453 datasets. Red areas indicate upregulated genes while the green areas indicate downregulated genes. 


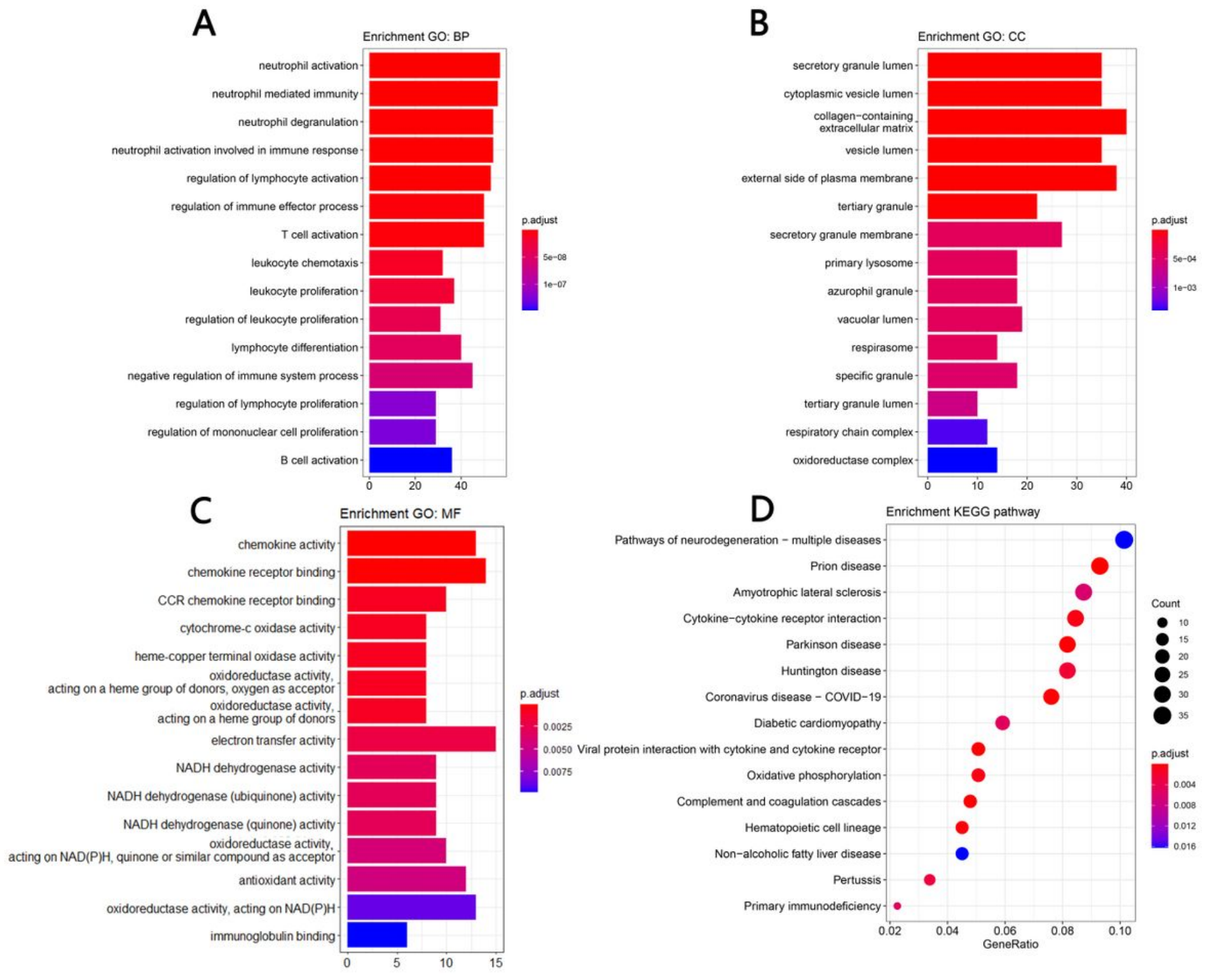

Figure 2

The top $15 \mathrm{GO}$ and KEGG pathways enrichment analysis of the differentially expressed genes. (A). Biological process; (B). Cellular component; (C). Molecular function; and (D). KEGG pathways. 


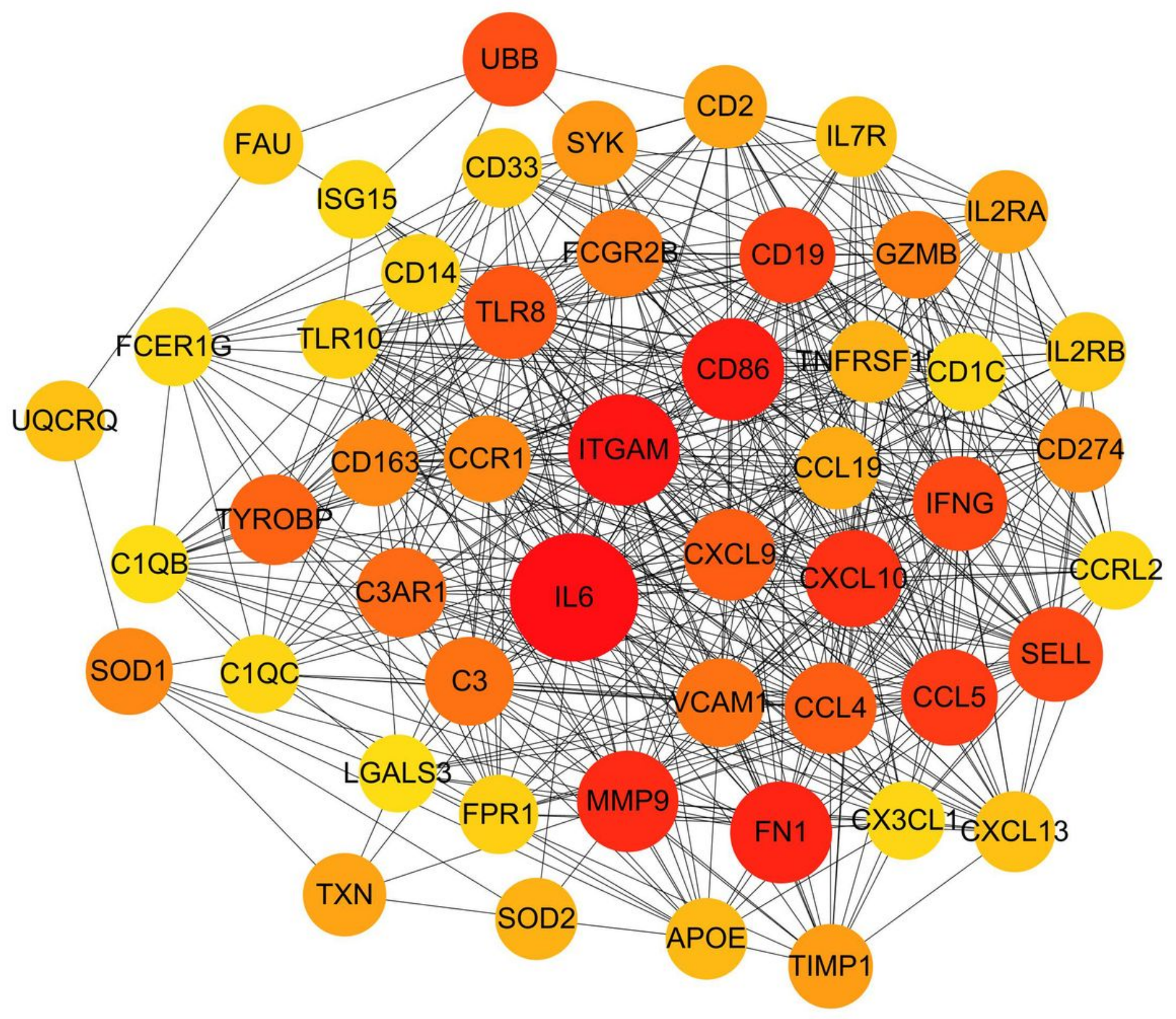

Figure 3

The protein-protein interaction network of the differentially expressed genes. The top 50 genes with the most node degree in the network visualized with cytoscape software. Node size is proportional to the degree of node degree. 


\section{Hub genes expression level in cHL cell lines}

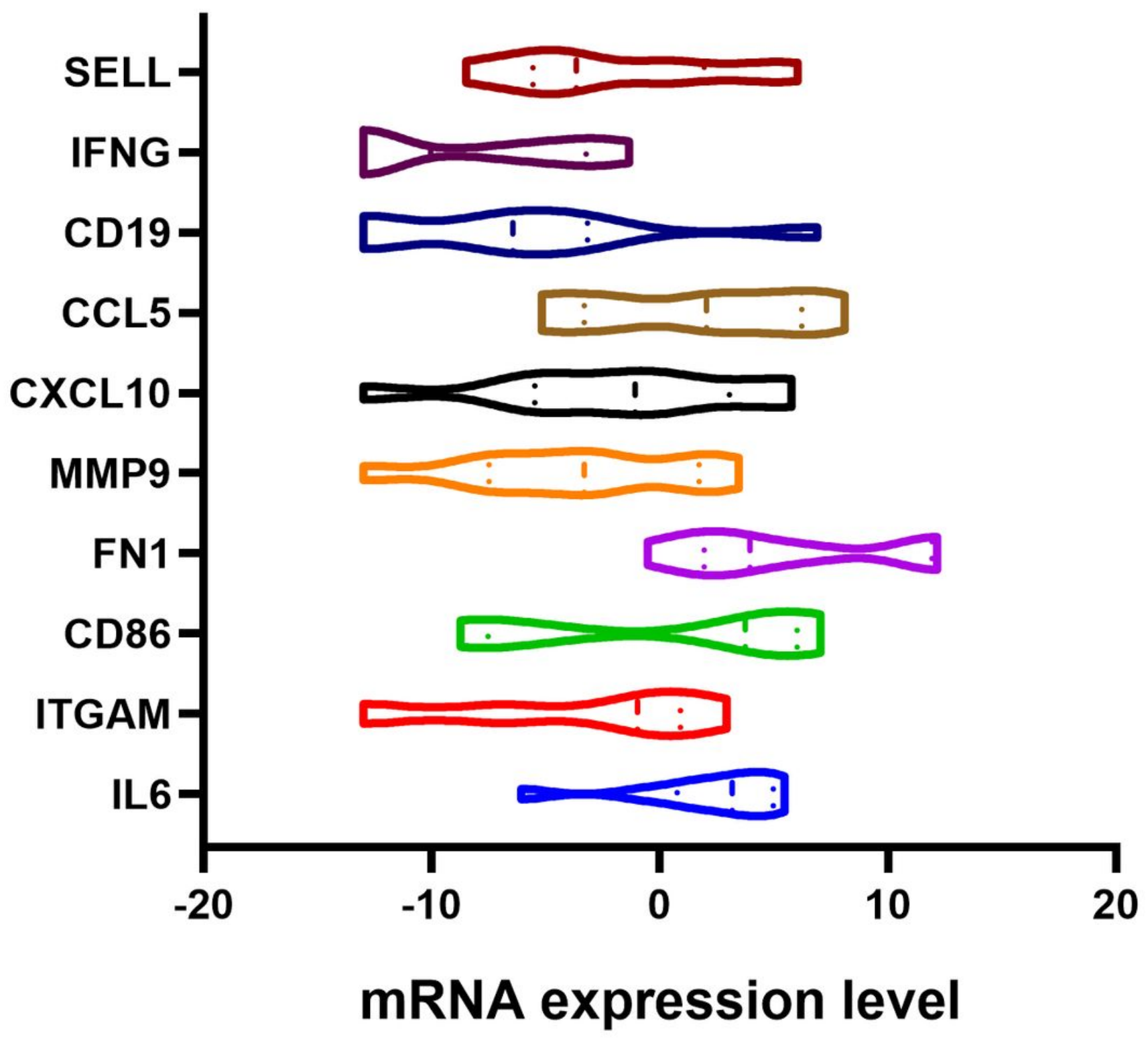

Figure 4

Validation of hub genes. The hub genes we imputed into the online database, the Cancer Cell Line Encyclopedia Cancer Cell Line Encyclopedia to verify the analysis results. The ordinate is the hub genes, and the abscissa is its mRNA expression. 